\title{
AVALIAÇÃO DAS ALTERAÇÕES ESTRUTURAIS EM UMA MEMBRANA ANIÔNICA CAUSADAS POR ENVELHECIMENTO EM CONTATO COM UM BANHO ELETROLÍTICO DE COBRE*
}

\author{
Juliana Mendonça Silva de Jesus ${ }^{1}$ \\ Tatiana Scarrazato ${ }^{2}$ \\ Jorge Alberto Soares Tenório ${ }^{3}$ \\ Denise Crocce Romano Espinosa ${ }^{4}$
}

\section{Resumo}

O estudo do comportamento de membranas trocadoras de íons em um sistema específico permite verificar a interação de um eletrólito com suas propriedades. No presente trabalho, foram utilizadas soluções de cobre à base de HEDP, proveniente de um banho galvânico isento de cianeto. A membrana utilizada foi a HDX 200 (aniônica) fornecida pela empresa Hidrodex. Amostras da membrana foram submetidas a um ensaio de envelhecimento durante 13, 26, 60 e 90 dias, imersas em uma solução a $5 \% \mathrm{v} / \mathrm{v}$ do banho de cobre. Após o envelhecimento essas foram preparadas para a realização de análises estruturais por FTIR, MEV e TGA. Os resultados obtidos pelo MEV e FTIR mostram que a membrana possui morfologia heterogênea. Além disso, verificou-se pelos resultados da análise térmica que o complexo presente no banho eletrolítico influi no comportamento térmico da membrana.

Palavras-chave: Análises estruturais; Membranas de troca iônica; Envelhecimento; Eletrodiálise.

\section{EVALUATION OF STRUCTURAL CHANGE IN A ANIONIC MEMBRANE CAUSED BY AGEING TEST WITH A BATH ELECTROLYTIC COPPER}

\section{Abstract}

The study behavior of ion exchange membranes in a specific system allows the verification of the interaction of an electrolyte with its properties. In this study, copper solutions were used based on HEDP, from a galvanic bath free of cyanide. The membrane used was the HDX 200 (anionic) provided by the company Hidrodex. Membrane samples were subjected to an ageing test for 13, 26, 60 and 90 days, immersed in a $5 \% \mathrm{v} / \mathrm{v}$ solution of copper bath. After ageing, these were prepared to perform structural analysis by FTIR, SEM and TGA. The results obtained by SEM and FTIR indicate that the membrane has a heterogeneous morphology. Furthermore, it was found from the results of thermal analysis that the complex present in the electrolytic bath affects the thermal behavior of the membrane.

Keywords: Structural analysis; lon-exchange membranes; Ageing test; Electrodialysis.

1 Química, Mestranda pelo Departamento de Engenharia Química da Escola Politécnica, Universidade de São Paulo (USP), São Paulo, São Paulo, Brasil.

2 Engenheira mecânica, mestre em ciências, doutoranda do Departamento de Engenharia Química, Escola Politécnica da Universidade de São Paulo, São Paulo, São Paulo, Brasil.

3 Engenheiro Metalurgista, doutor em engenharia metalúrgica, professor titular, Departamento de Engenharia Química, Escola Politécnica da Universidade de São Paulo, São Paulo, São Paulo, Brasil.

4 Engenheira Metalurgista, doutora em engenharia metalúrgica, professora associada, Departamento de Engenharia Química, Escola Politécnica da Universidade de São Paulo, São Paulo, São Paulo, Brasil. 


\section{INTRODUÇÃO}

A aplicação de membranas trocadoras de íons em processos de separação como a eletrodiálise, tem se difundido em áreas industriais. Com o foco, de promover a redução de emissão de efluentes, de impactos ambientais e na recuperação de insumos [1].

O uso de novas tecnologias no tratamento de efluentes tem-se destacado, juntamente com o avanço na formulação de banhos galvânicos. Um exemplo deste avanço, é a substituição do cianeto, a fim de reduzir a toxicidade do processo e de seus resíduos gerados. 01 hidroxietano- 1,1 difosfônico, ou HEDP, é um ácido orgânico que possui caráter complexante e tem sido utilizado em banhos de cobre alcalino para substituir o cianeto (Tabela 1) [2].

Segundo Vargas [2], o HEDP apresentou eficiência no processo de eletrodeposição, mostrando equivalência ao cianeto para tal função. Contudo, esse novo componente possui custo comercial superior ao cianeto, sendo assim necessário a aplicação de uma tecnologia de tratamento de efluentes que possa recuperar o HEDP, tornando o processo sustentável e aplicável [3].

Tabela 1 - Parâmetros para a preparação de um banho de cobre a base de HEDP [2].

\begin{tabular}{cc}
\hline COMPOSIÇÃO & CONCENTRAÇÃO BASE \\
\hline lons $\mathrm{Cu}^{2+}$ & $4,5 \mathrm{~g} \cdot \mathrm{L}^{-1}$ \\
\hline $\mathrm{HEDP}$ & $105 \mathrm{~g} \cdot \mathrm{L}^{-1}$ \\
\hline Cloreto de Potássio & $7.0 \mathrm{~g} \cdot \mathrm{L}^{-1}$ \\
\hline $\mathrm{pH}$ & $\sim 10,0$ \\
\hline
\end{tabular}

Assim, a utilização da eletrodiálise foi proposta em um estudo que obteve extrações percentuais de cerca de $99,7 \%$ de cobre e $94,4 \%$ de HEDP [3].

As membranas de troca iônica possuem propriedades como a permeseletividade, a estabilidade térmica, a resistência elétrica, o grau de inchamento, a capacidade de troca iônica. Essas propriedades são responsáveis pela definição e caracterização das membranas, além de serem aspectos influentes na escolha das membranas para os processos industriais [4,5].

Em relação à estrutura as membranas trocadoras de íons possuem uma matriz polimérica e cargas elétricas anexadas em seus interstícios (Figura 1). As membranas podem ser classificadas em catiônica e aniônica,. São frequentes também a distinção entre homogênea e heterogênea, em função da forma em que a resina trocadora de íons foi distribuída sobre a matriz polimérica em seu processo de fabricação $[1,4,5]$. 
Legenda:

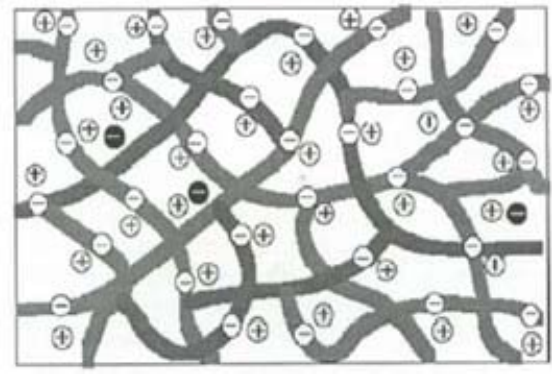

lons fixos (-)

- Contra-ions (+)

- Co-ion (-)

- Matriz polimérica

Figura 1 - Esquema da estrutura de uma membrana catiônica (Adaptado) [1].

Em geral, as membranas trocadoras de íons são produtos poliméricos e sintéticos, de composição final variada. Mulder[5], cita os polieletrólitos que são polímeros constituídos de grupos iônicos com cargas que diferencia sua classificação. Um exemplo disso, é as cadeias resultantes da polimerização do poliestireno e do divinilbenzeno, que foram adicionados grupos de cargas distintas, caracterizando em polímeros passíveis de utilização em membranas aniônicas e catiônicas. Há grupos funcionais de uso comum como os sais de amônio para membranas aniônicas e grupos sulfônicos ou ácidos carboxílicos usuais para membranas catiônicas.

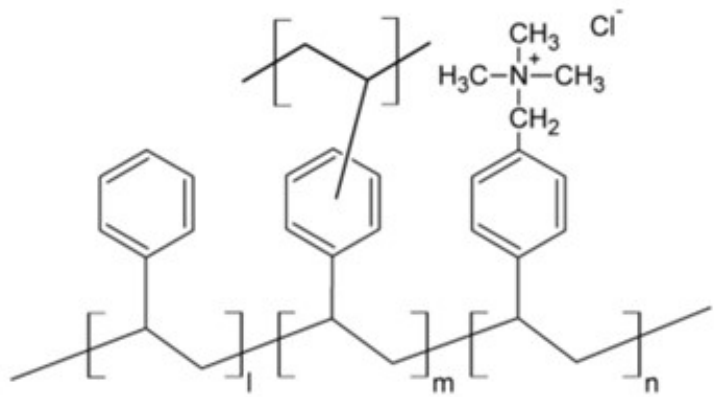

Figura 2 - Esquema da estrutura do poliestireno ligado ao divinilbenzeno e a grupos trimetil de amônio [6].

A estabilidade térmica da membrana é uma propriedade avaliada a partir de ensaios termogravimétricos, uma vez que a incorporação iônica pode afetar o comportamento térmico das membranas e ocasionar alterações estruturais[7].

O teste de envelhecimento tem como objetivo avaliar tais propriedades em função do tempo e do meio que as membranas são submetidas. Além disso, as membranas possuem uma vida útil, que pode variar em função do tempo de contato com o meio de trabalho aplicado. Assim, o envelhecimento também pode indicar do efeito de possíveis incorporações e degradações das membranas em um determinado meio [8]. Técnicas espectroscópicas, como o FTIR, têm sido utilizadas para caracterizar as principais ligações existentes nas membranas sintéticas, fornecendo informações adicionais sobre os materiais que as compõem. A partir disso, pode-se distinguir por meio de um espectro de infravermelho, se a membrana é homogênea ou heterogênea, entre outras informações e apontar os grupos funcionais [6]. 


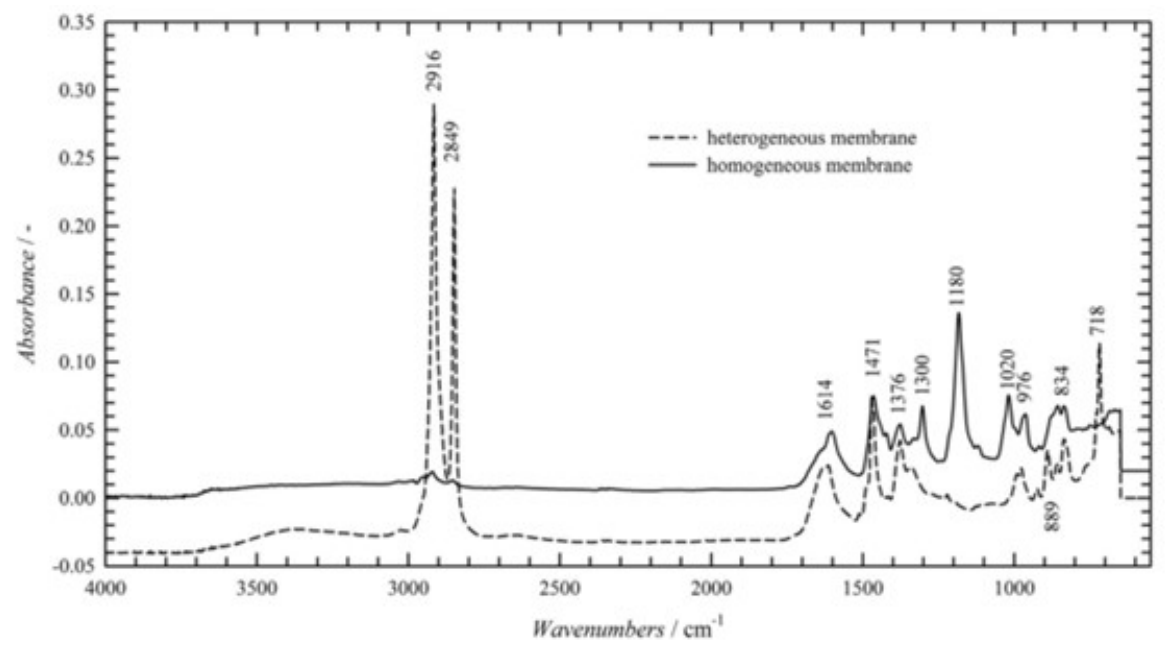

Figura 3 - FTIR de uma membrana homogênea e heterogênea produzida em laboratório [6].

Demais técnicas, como a microscopia eletrônica de varredura (MEV), e a análise termogravimétrica são ferramentas usuais para a caracterização de membranas. $O$ presente estudo tem como objetivo avaliar o efeito da incorporação de íons provenientes de um banho galvânico de cobre à base de HEDP, na estabilidade térmica de membranas sintéticas de troca iônica. Os parâmetros de comparação avaliados na discussão dos resultados são os resultados das análises de FTIR, MEV e TGA da membrana nova, em relação ás envelhecidas.

\section{MATERIAIS E MÉTODOS}

A Figura 4 mostra as etapas realizadas para este estudo, onde a partir do banho de cobre à base de HEDP, foi realizado uma diluição deste para uma concentração de $5 \% \mathrm{v} / \mathrm{v}$ em volume do banho original.

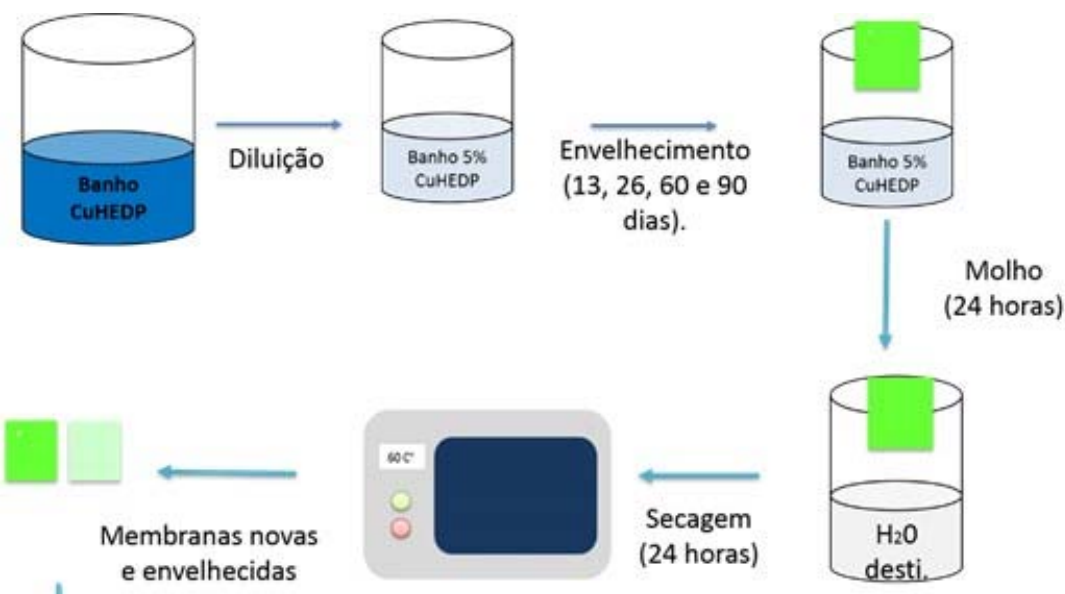

\section{ANÁLISES ESTRUTURAIS}

TGA

MEV

FTIR

Figura 4 - Esquema da sequência de ensaios realizados para este estudo. 
A membrana selecionadas para o estudo foi a HDX 200 (aniônica) (Figura 5), que foi submetida a testes de envelhecimento na solução diluída a $5 \% \mathrm{v} / \mathrm{v}$ por um período de $13,26,60$ e 90 dias.

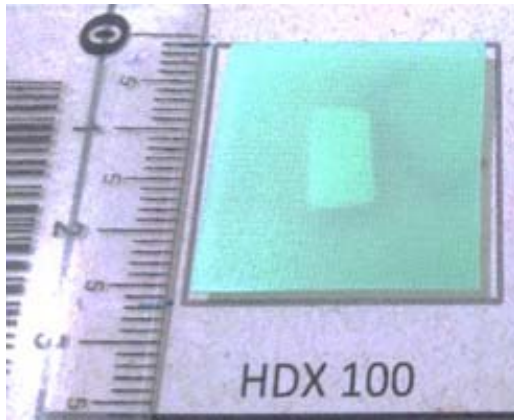

Figura 5 - Membrana HDX 200 nova.

Como demonstrado pela Figura 4, após o termino do envelhecimento, as membranas foram lavadas em água destilada por 24 horas, a fim de remover qualquer impureza superficial. Em seguida foram secas em estufa à $60^{\circ} \mathrm{C}$ por 24 horas. Por fim, as membranas foram encaminhadas para análises estruturais usando as técnicas de MEV, FTIR e ao ensaio termogravimétrico (NETZSCH STA 449F1). Para este, utilizouse uma razão de aquecimento foi de $20^{\circ} \mathrm{C}$ por minuto sob atmosfera de nitrogênio, $\mathrm{o}$ termino do teste deu-se a $1000^{\circ} \mathrm{C}$,

Ressalta-se que essas técnicas foram aplicadas nesse estudo com a finalidade de avaliar possíveis sinais de incorporação e degradação das membranas aniônicas em contato com o complexo CuHEDP.

\section{RESULTADOS E DISCUSSÃO}

\subsection{Envelhecimento}

A Figura 6 representa as membranas após o termino do teste de envelhecimento. Notase que as amostras apresentaram alterações na sua coloração, em comparação com seu estado inicial (Figura 5).
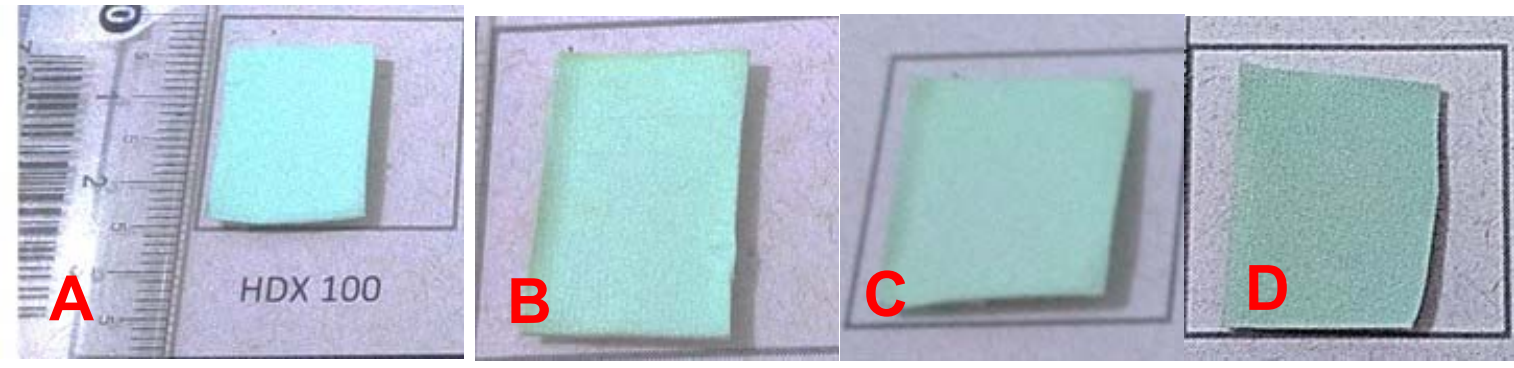

Figura 6 - Membranas HDX 200 após o teste de envelhecimento (a)13 dias, (b) 26 dias, (c) 60 dias e (d) 90 dias.

\subsection{Análises estruturais}

O MEV realizado das membranas envelhecidas apresenta a aparência visual de uma uma morfologia heterogênea. Como citado anteriormente, e que não apresentaram alterações visuais relacionas à incorporação iônica e degradação (Figura 7) . 

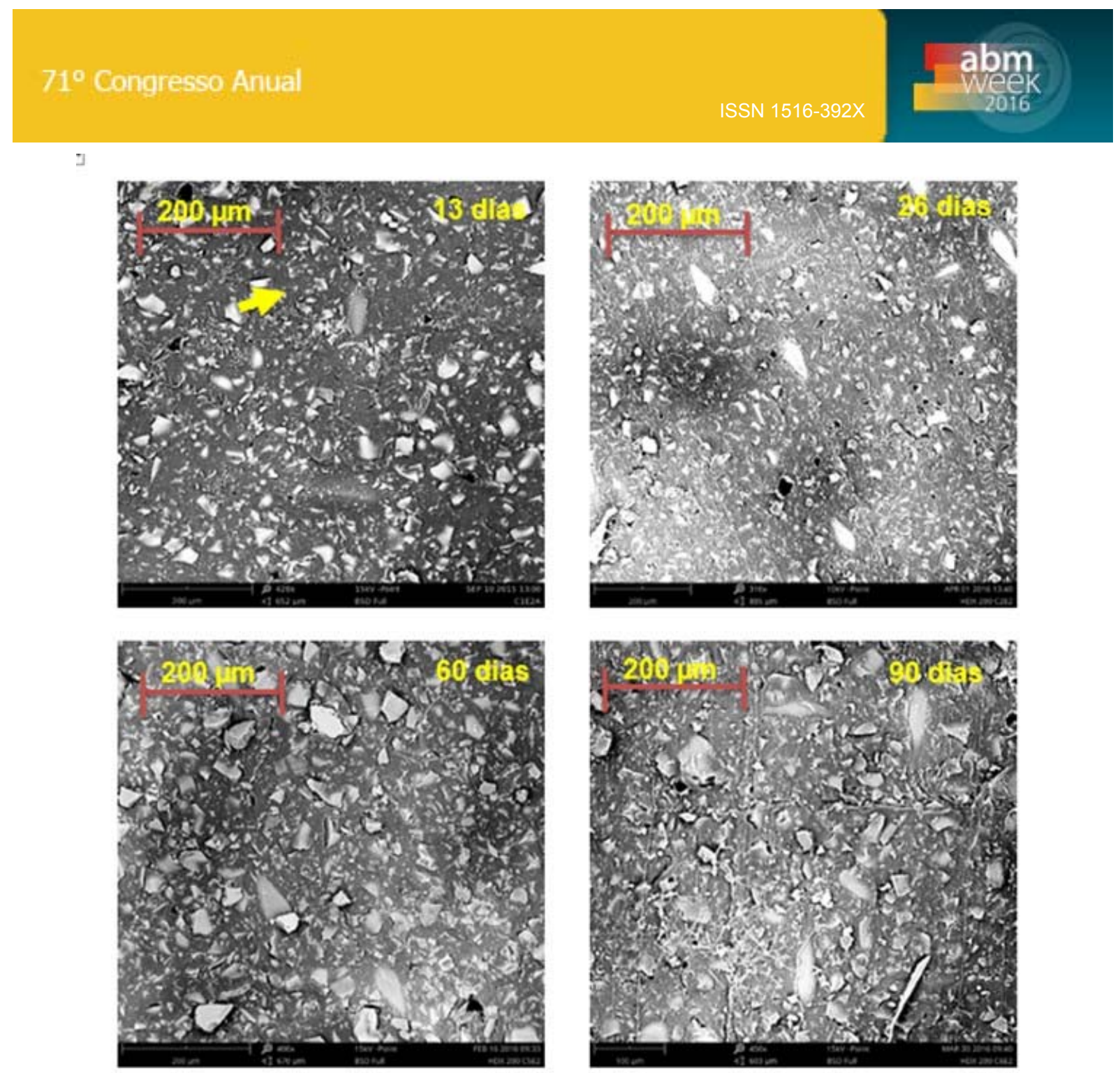

Figura 7 - Imagem de elétrons retroespalhados obtida por MEV membrana HDX 200 envelhecidas. A área indicada pela seta amarela refere-se ao reforço estrutural da matriz polimérica.

A Figura 8 apresenta a análise termogravimétrica das membranas HDX 200 em seu estado inicial, ou seja, nova, e as membranas envelhecidas em solução de CuHEDP $5 \%$ durante 13, 26, 60 e 90 dias. Além disso foi analisada primeira derivada (DTG) da membrana HDX 200 nova para a distinção das regiões onde ocorrem reações referentes à degradação térmica. A partir desse resultado, pode-se notar que as membranas envelhecidas apresentaram um aumento na perda de massa na inflexão que ocorreu entre 600 a $1000 \mathrm{C}^{\circ}$. A membrana nova apresentou, nessa mesma faixa, um decréscimo de massa de $100 \%$, mostrando que houve total degradação. Para membranas envelhecidas, houve resíduos de degradação entre 4,0 a 10,0 g, sendo esses valores proporcionais ao tempo de contato, indicando que houve incorporação de compostos inorgânicos nas membranas. Além disso, nota-se que o comportamento térmico das membranas envelhecidas foi distinto da membrana nova. A DTG da membrana nova mostrou três picos de reação, enquanto a TGA das envelhecidas não apresentaram esses picos (Figura 9). 
HDX 200 - CUHEDP 5\%

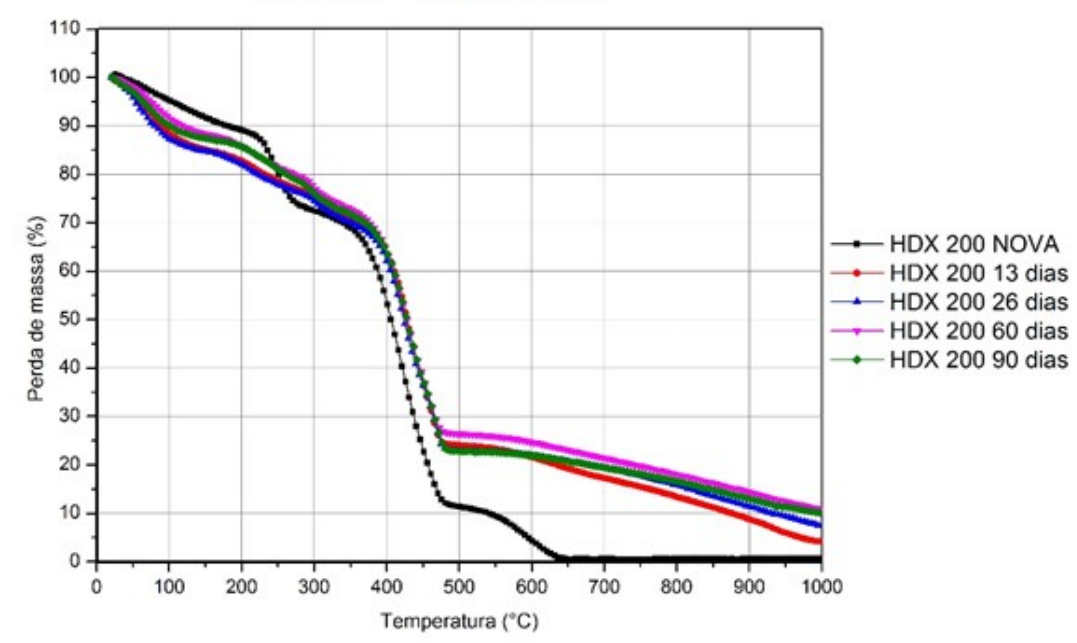

Figura 8 - TGA das membranas HDX 200 imersas em banho de CuHEDP a $5 \%$ e da membrana HDX 200 nova.

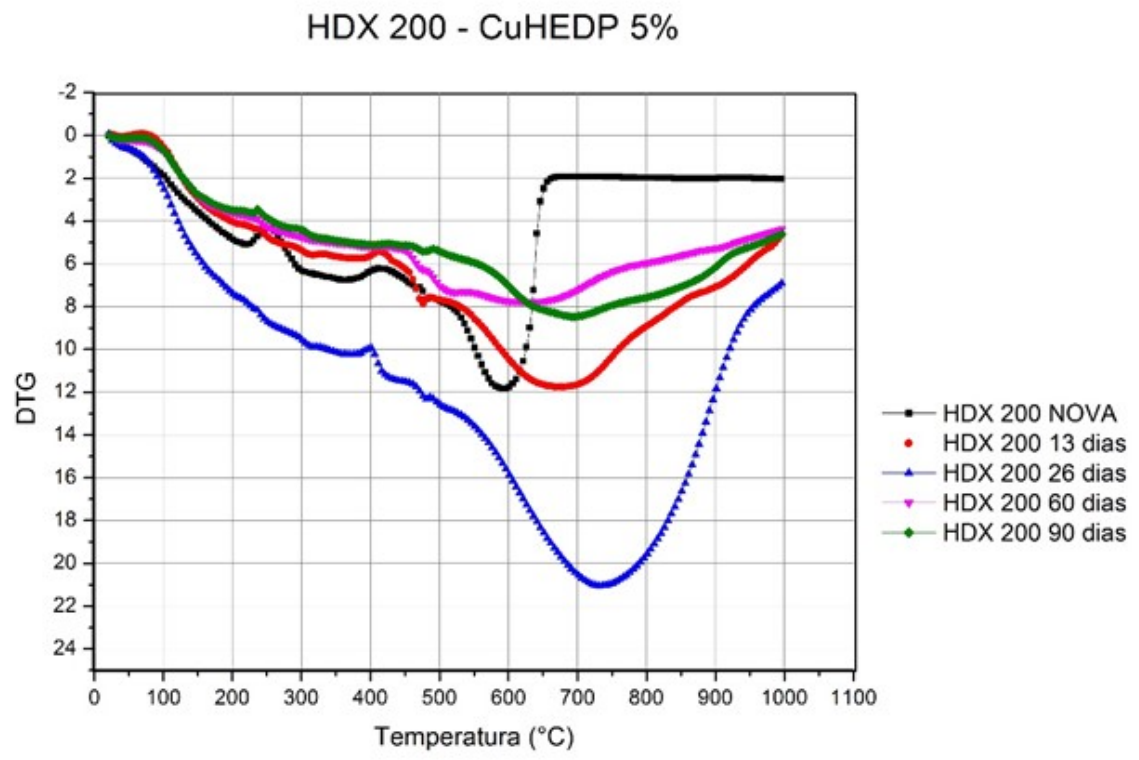

Figura 9 - DTG das membranas HDX 200 imersas em banho de CuHEDP a 5\% e da membrana HDX 200 nova.

Os espectros obtidos pela análise de FTIR (Figura 10) representou um comportamento morfológico de uma membrana heterogênea, quando comparada com a obtida nos realizados por Karas et. Al [6]. Além disso, informações a respeito do grupo funcional podem ser obtidos pelos picos de frequência 1471 e 1376, que são provenientes da vibração das ligações entre $\mathrm{NH}_{2}{ }^{+}-\mathrm{CH}_{3}$ e $\mathrm{NH}^{+}\left(\mathrm{CH}_{3}\right)_{3} \mathrm{Cl}^{-}$. Esses grupamentos aminas possuem caráter de base fraca com valores de pka entre 6 a 10 [6]. Adicionalmente, os espectros apresentam o aparecimento de novos picos em relação ao espectro da membrana nova, sendo esses provenientes da interação iônica. 

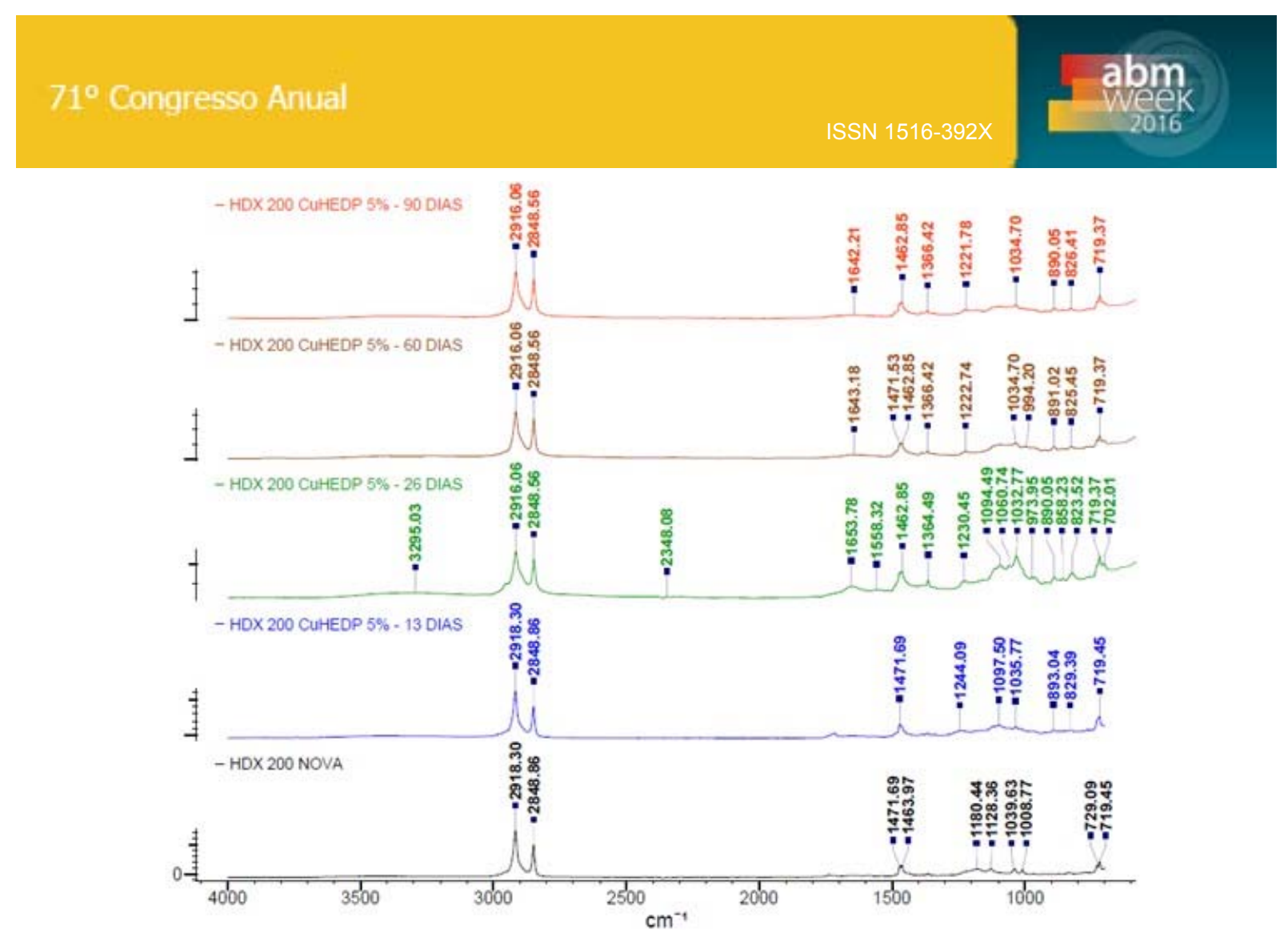

Figura 10 - FTIR das membranas HDX 200 imersas em banho de CuHEDP a 5\%.

\section{CONCLUSÃO}

Os ensaios de envelhecimento foram realizados a fim de avaliar a influência do complexo de CuHEDP na membrana aniônica e heterogênea por um período total de 2160 horas de contato. Também foram avaliadas as propriedades estruturais como a morfologia, grupos funcionais e o comportamento térmico.

Análises de MEV demonstraram a distribuição não uniforme da resina na matriz polimérica caracterizando-a como heterogênea. Além disso, as membranas analisadas por MEV e que foram envelhecidas não apresentaram modificações visuais e sinais de incorporação.

A análise termogravimétrica foi utilizada para a verificação do comportamento térmico em relação à incorporação iônica. As membranas envelhecidas demonstram comportamento distinto da membrana nova, como o percentual de perda de massa entre 600 a $1000 \mathrm{C}^{\circ}$. A variação da massa residual das membranas envelhecidas, também demonstraram crescente acréscimo em relação ao tempo de contato, sendo que o maior tempo de contato resultou em uma maior massa residual

O FTIR mostrou que a membrana aniônica possui grupos aminas sendo esses caracterizados como bases fracas. Além disso, notou-se a influência do contato com o complexo CuHEDP e as membranas, por meio do aparecimento de novos picos em relação a membrana nova.

Os resultados obtidos indicaram que o complexo presente no banho de cobre alcalino exerce influências e alterações estruturais sob a membrana, embora não ter sido observado sinas de degradação. 


\section{REFERÊNCIAS}

1 Strathmann, H. Ion-exchange membrane separation processes. Amsterdam: Elsevier,2004.

2 Vargas, C. Estudo da eletrodeposição do cobre a partir de banhos alcalinos isentos de cianetos. 2008, 221 p. Dissertação (Mestrado em Engenharia) - Escola

Politécnica,Universidade de São Paulo, São Paulo, 2008.

3 Scarazzato, T. Tratamento de efluentes contendo HEDP por eletrodiálise. 2013. Dissertação (Mestrado em Engenharia) - Escola Politécnica,Universidade de São Paulo, São Paulo, 2013.

4 Sata T. Ion Exchange Membranes: Preparation, Characterization, Modification and Application. In 2004.

5 Mmulder, M. Basic principles of membrane technology. Segunda edição. Enschede, The Netherlands: Kluwe Academic, 1996.

6 Karas, F; Hnát, J; Paidar, M, Schauer, J; Bouzek, K. Determination of the ion-exchange capacity of anion-selective membrane. International Journal of Hydrogen Energy. 2014; 39:5054-62.

7 Wu L, Pan Q, Varcoe JR, Zhou D, Ran J, Yang Z, et al. Thermal crosslinking of an alkaline anion exchange membrane bearing unsaturated side chains. Journal of Membrane Science. 2015; 409:1-8.

8 Garcia-Vasquez W, Ghalloussi R, Dammak L, Larchet C, Nikonenko V, Grande D. Structure and properties of heterogeneous and homogeneous ion-exchange membranes subjected to ageing in sodium hypochlorite. Journal of Membrane Science. 2014. 452:104-16. 